on growth character and impact on the heart of the offspring subject is still subject to discussion. $\mathrm{N}$-cadherin plays a critical role in myocardial development and function and is associated with the development of heart failure. Studies have shown a nicotine receptor mediated pathway was associated with the modulation of $\mathrm{N}$-cadherin expression. In summary, we hypothesise that prenatal treatment of nicotine might alter myocardial $\mathrm{N}$-cadherin expression which may predispose for impaired cardiac function in later life.

Methods Sprague-Dawley rats were infused with nicotine (12 $\mathrm{mg} / \mathrm{kg} / \mathrm{day})$ or saline via subcutaneous osmotic minipumps throughout the gestation. We weighed brain, heart, lung, liver, and kidney of one randomly selected pup of each litter within 3-12 h after birth. The litter size was then reduced to eight pups to ensure equal nutrient access. Body weight was measured every 2 days after birth. Heart samples from the offspring at birth and 45 days of age were serially sliced for measuring $\mathrm{N}$-cadherin by Immuno-histochemistry staining and analysed by western blot for $\mathrm{N}$-cadherin expression.

Results The birth weights of prenatal nicotine exposure offspring were significantly reduced compared with control offspring $(6.72 \pm 0.38 \mathrm{~g}$ vs $7.40 \pm 0.35 ;$ p 0.05$)$. Brain weight-to-body weight was significantly higher, and the liver weight-to-body weight ratio was prominently lower in maternal hypoxia offspring compared with control, indicating a relative increased head circumference and decreased abdominal circumference at birth. But there were no obvious differences in mean weight ratios of heart, lung, and kidney. N-cadherin was expressed in both atrium and ventriculium, predominantly localised to adherent junctions in intercalated discs. There was a significant down-regulated protein expression of $\mathrm{N}$-cadherin in prenatal nicotine exposure offspring when compare with control.

Conclusions Prenatal nicotine exposure resulted in intrauterine growth restriction with disproportion in neonatal organ size as a sign of relative increased head circumference and decreased abdominal circumference at birth of offspring who exhibited a postnatal catch-up growth. These growth characters are associated with a higher risk of cardiovascular disease. Furthermore, we demonstrate a down-regulated protein expression of $\mathrm{N}$-cadherin in the myocardium from prenatal nicotine exposure offspring, which might predispose for impaired cardiac function in later life.

\section{[gw22-e0741] PRENATAL NICOTINE EXPOSURE MODULATES FETAL GROWTH AND REDUCES MYOCARDIAL N-CADHERIN EXPRESSION}

Wang Zhenhua, Huang Ziyang Second Affiliated Hospital Of Fujian Medical University, Fujian, China

10.1136/heartjnl-2011-300867.234

Objectives To investigate the impact of prenatal nicotine exposure on the birth weight, body shape at birth and postnatal growth of the offspring, and the expression of myocardial $\mathrm{N}$-cadherin.

Background Epidemiological studies have demonstrated that low birth weight, relative increased head circumference, and decreased abdominal circumference at birth, and postnatal catch-up growth by the suboptimal intrauterine environment are associated with increased risk of death from cardiovascular disease. The influence of prenatal nicotine exposure 